Article

\title{
Dual MIMU Pedestrian Navigation by Inequality Constraint
}

\section{Kalman Filtering}

\author{
Wei Shi ${ }^{1}$, Yang Wang ${ }^{1, *}$ and Yuanxin $\mathrm{Wu}^{2}$ \\ 1 School of Aeronautics and Astronautics, Central South University, Changsha 410083, China; ahshw@csu.edu.cn \\ 2 Shanghai Key Laboratory of Navigation and LocationbasedServices, School of Electronic Information and Electrical Engineering, \\ Shanghai Jiao Tong University, Shanghai 200240, China; yuanx_wu@hotmail.com \\ * Correspondence: sunshineofking@163.com; Tel.:+86-731-8887-7246
}

\begin{abstract}
The foot-mounted inertial navigation system is an important application of pedestrian navigation as it in principle does not rely any external assistance. A real-time range decomposition constraint method is proposed in this paper to combine the information of dual foot-mounted inertial navigation systems. It is well known that low-cost inertial sensors with ZUPT (zero-velocity update) and range decomposition constraint perform better than in either single way. This paper recommends that the distance of separation between the position estimates of feet-mounted inertial navigation systems be restricted in the ellipsoidal constraint which relates to the maximum step and leg height. The performance of the proposed method is studied utilizing experimental data. The results indicate that the method can effectively correct the dual navigation systems' position over the existing spherical constraint.
\end{abstract}

Keywords: inertial navigation system; ZUPT; ellipsoidal constraint; correct position

\section{Introduction}

Positioning and tracking systems have developed for several decades in various applications ranging from tracking of pedestrians to autonomous vehicles [1]. A high-precision navigation system is often needed for urban and indoor lives where the GPS is unavailable, for example in wearable body area network [2]. An increasing attention for the pedestrian navigation problem can be partially attributed to a great progress in affordable wearable computing platforms and enhancement in sensor quality, especially the Micro Electro Mechanical Systems (MEMS) [3]. The Micro Inertial Measurement Units (MIMU) usually consists of accelerometers, gyroscopes, magnetometers and pressure sensors [4].

In real life application, however, the low-cost inertial navigation systems suffer from the accumulation of errors while calculating the traveled distance of the objects. These errors let the trajectories drift away from the actual path as time grows. An effective technique to bound the error growth is using Zero Velocity Update (ZUPT) [5]. The foot-mounted ZUPT-aided INS was provided as an open source in Openshoe project [6].

When we use the ZUPT-aided INS to track pedestrian location, the heading drift of navigation information is unobservable [7], so it is difficult to get accurate location information only through ZUPT in long time navigation. Some previous works used the information fusion of multiple inertial sensors to ulteriorly correct position [8-9]. As shown in [8], the use of the two feet range constraint can significantly improve the navigation performance and a 110-meter straight line experiment showed that the spherical constraint algorithm can reduce the mean error and covariance of the final position estimates.

In [9], the authors proposed a method for a dual-mounted INS to reduce the systematic heading drift. They set up a dual MIMU system with two feet, in which the maximum distance between the two systems is derived from the calibration procedure. The proposed algorithm can obtain the walk trajectory when the initial heading estimates are known, but the method did not significantly improve the positioning accuracy of the system.

The experiments in [8-9] are two dimensional (2-D) plane experiment, and the feasibility of the algorithm in three dimensional space was not verified. When we use the spherical constraint [8] based on maximum step size to correct the position of the two feet, if the step size is greater than the height of heels, the spherical constraint algorithm would have little significant effect on the height constraint of the two feet. Therefore, in this paper we take into account different separation constraints in level and height directions, which is shown to be more effective to correct the pedestrian location.

The outline of the paper is as follows: In Section II, we briefly describe the basic theory of the Kalman filter, and analyze the special form with inequality state constraints. In Section III, we propose the ellipsoidal constraint method that fuses the navigation information from two MIMUs mounted on pedestrian feet. In contrast with previous works, the method uses physiological characteristics of the two feet's maximum separation, and decomposes the step constraint further into three degrees of freedom. In Section IV, we implement two experiments to verify the effectiveness of the proposed method. Finally, conclusions are presented in Section V. 


\section{Principle and theory}

\subsection{Discrete Kalman Filter}

The Kalman filter addresses the general problem of estimating the state $\boldsymbol{X}_{k}$ of a discrete-time process that is governed by the linear stochastic difference equation [10][11].

$$
\boldsymbol{X}_{k}=\boldsymbol{\Phi}_{k, k-1} \boldsymbol{X}_{k-1}+\boldsymbol{\Gamma}_{k-1} \boldsymbol{W}_{k-1}
$$

with a measurement $\boldsymbol{Z}_{k}$ that is

$$
\boldsymbol{Z}_{k}=\boldsymbol{H}_{k} \boldsymbol{X}_{k}+\boldsymbol{V}_{k}
$$

Where $\boldsymbol{\Phi}_{k, k-1}$ denotes transition matrix relating the state at the previous time step $t_{k-1}$ to the state at the current step $t_{k}, \boldsymbol{\Gamma}_{k-1}$ denotes the system noise drive matrix, $\boldsymbol{H}_{k}$ represents a measurement matrix, $\boldsymbol{V}_{k}$ is series of measurement noise, $\boldsymbol{W}_{k}$ represents noise excitation sequence for the system. The $\boldsymbol{W}_{k}$ and $\boldsymbol{V}_{k}$ simultaneously meet:

$$
\left.\begin{array}{l}
E\left[\boldsymbol{W}_{k}\right]=\mathbf{0}, \operatorname{Cov}\left[\boldsymbol{W}_{k}, \boldsymbol{W}_{j}\right]=E\left[\boldsymbol{W}_{k} \boldsymbol{W}_{j}^{\mathrm{T}}\right]=\boldsymbol{Q}_{k} \delta_{k j} \\
E\left[\boldsymbol{V}_{k}\right]=\mathbf{0}, \operatorname{Cov}\left[\boldsymbol{V}_{k}, \boldsymbol{V}_{j}\right]=E\left[\boldsymbol{V}_{k} \boldsymbol{V}_{j}^{\mathrm{T}}\right]=\boldsymbol{R}_{k} \delta_{k j} \\
\operatorname{Cov}\left[\boldsymbol{W}_{k}, \boldsymbol{V}_{j}\right]=E\left[\boldsymbol{W}_{k} \boldsymbol{V}_{j}^{\mathrm{T}}\right]=\mathbf{0}
\end{array}\right\}
$$

In practice, the process noise covariance matrices $\boldsymbol{Q}_{k}$ and measurement noise covariance matrices $\boldsymbol{R}_{k}$ might change with each time step or measurement, and we assume they are positive definite matrix.

$$
\begin{gathered}
\hat{\boldsymbol{X}}_{k}=\hat{\boldsymbol{X}}_{k / k-1}+\boldsymbol{K}_{k}\left(\boldsymbol{Z}_{k}-\boldsymbol{H}_{k} \hat{\boldsymbol{X}}_{k / k-1}\right) \\
\boldsymbol{K}_{k}=\boldsymbol{P}_{k / k-1} \boldsymbol{H}_{k}^{\mathrm{T}}\left(\boldsymbol{H}_{k} \boldsymbol{P}_{k / k-1} \boldsymbol{H}_{k}^{\mathrm{T}}+\boldsymbol{R}_{k}\right)^{-1} \\
\boldsymbol{P}_{k / k-1}=\boldsymbol{\Phi}_{k, k-1} \boldsymbol{P}_{k-1} \boldsymbol{\Phi}_{k, k-1}^{\mathrm{T}}+\boldsymbol{\Gamma}_{k-1} \boldsymbol{Q}_{k-1} \boldsymbol{\Gamma}_{k-1}^{\mathrm{T}} \\
\boldsymbol{P}_{k}=\left(\boldsymbol{I}-\boldsymbol{K}_{k} \boldsymbol{H}_{k}\right) \boldsymbol{P}_{k / k-1}
\end{gathered}
$$

The formulas (4)-(7) are the basic equations of Kalman filtering. If the initial values about $\hat{\boldsymbol{X}}_{0}$ and $\boldsymbol{P}_{0}$ are given, we can perform the state estimation at time $k$ according to measurements $\boldsymbol{Z}_{k}$ at the same moment.

\subsection{Inequality Kalman Filter}

The inequality Kalman filter appears in solving practical problems between state variables where there are inequality relationships [12]. The inequality relationship can be expressed as a constraint equation and combined with the Kalman filter, as a result of which the optimal solution strictly conforms to the inequality constraints between state variables, and a better result could be obtained.

The inequality-constrained Kalman optimal solution [13] is expressed as:

$$
\left.\begin{array}{l}
\min _{\hat{\boldsymbol{x}}}\left(\hat{\boldsymbol{x}}_{k}-\boldsymbol{x}_{k}\right)^{\mathrm{T}} \mathbf{T}\left(\hat{\boldsymbol{x}}_{k}-\boldsymbol{x}_{k}\right) \\
\boldsymbol{L} \boldsymbol{x}_{k} \leq \boldsymbol{d}
\end{array}\right\}
$$

where $\boldsymbol{x}_{k}$ is the unconstrained (standard) Kalman filter estimate and $\mathbf{T}$ is a symmetric positive definite weighting matrix [14-15]. As such, the weighted error of the constrained filter is minimized [16].

Expanding the first type of (8)

$$
\left(\hat{\boldsymbol{x}}_{k}-\boldsymbol{x}_{k}\right)^{\mathrm{T}} \mathbf{T}\left(\hat{\boldsymbol{x}}_{k}-\boldsymbol{x}_{k}\right)=\hat{\boldsymbol{x}}_{k}^{\mathrm{T}} \mathbf{T} \hat{\boldsymbol{x}}_{k}-2 \boldsymbol{x}_{k}^{\mathrm{T}} \mathbf{T} \hat{\boldsymbol{x}}_{k}+\boldsymbol{x}_{k}^{\mathrm{T}} \mathbf{T} \boldsymbol{x}_{k}
$$

Thus, the inequality constrained problem can be further simplified as:

$$
\left.\begin{array}{l}
\min _{\hat{\boldsymbol{x}}}\left(\hat{\boldsymbol{x}}_{k}^{\mathrm{T}} \mathbf{T} \hat{\boldsymbol{x}}_{k}-2 \boldsymbol{x}_{k}^{\mathrm{T}} \mathbf{T} \hat{\boldsymbol{x}}_{k}\right) \\
\boldsymbol{L} \boldsymbol{x}_{k} \leq \boldsymbol{d}
\end{array}\right\}
$$

\section{Methods}

3.1 Generalized Likelihood Ratio Test (GLRT)

The output of MIMU can be expressed as

$$
\boldsymbol{x}_{k}=\left[\begin{array}{ll}
\boldsymbol{x}_{k}^{a} & \boldsymbol{x}_{k}^{\omega}
\end{array}\right]^{\mathrm{T}}
$$

where the specific force measurement vector $\boldsymbol{x}_{k}^{a} \in \Omega^{3}$ and the angular rate measurements vector $\boldsymbol{x}_{k}^{\omega} \in \Omega^{3}$. Assuming a series of measured value $\boldsymbol{y}_{n}=\left\{\boldsymbol{x}_{k}\right\}_{k=n}^{n+N-1}$. We employ a double hypothesis testing as such, $\mathrm{H}_{0}:$ MIMU stationary, $\mathrm{H}_{1}:$ MIMU 
moving. The false alarm probability is expressed as:

$$
P_{F A}=P\left\{\mathrm{H}_{0} \mid \mathrm{H}_{1}\right\}=\alpha
$$

The detection probability is $P_{D}=P\left\{\mathrm{H}_{0} \mid \mathrm{H}_{0}\right\}$. Two hypotheses observation data probability density function are respectively defined as $p\left(\boldsymbol{y}_{n} ; \mathrm{H}_{0}\right)$ and $p\left(\boldsymbol{y}_{n} ; \mathrm{H}_{1}\right)$.

The mathematical sensor model can be expressed as: $\boldsymbol{x}_{k}=\boldsymbol{s}_{k}(\theta)+\boldsymbol{v}_{k}$, where $\boldsymbol{s}_{k}(\theta)=\left[\boldsymbol{s}_{k}^{a}(\theta) \quad \boldsymbol{s}_{k}^{\omega}(\theta)\right]^{\mathrm{T}}$ and $\boldsymbol{v}_{k}=\left[\begin{array}{ll}\boldsymbol{v}_{k}^{a} & \boldsymbol{v}_{k}^{\omega}\end{array}\right]^{\mathrm{T}}$, the force of MIMU is $\boldsymbol{s}_{k}^{a}(\theta) \in \Omega^{3}$, MIMU angular rate is expressed as $\boldsymbol{s}_{k}^{\omega}(\theta) \in \Omega^{3}$. The symbol $\theta$ denotes the vector of unknown elements $\boldsymbol{v}_{k}^{a} \in \Omega^{3}$ accelerometers noise, $\boldsymbol{v}_{k}^{\omega} \in \Omega^{3}$ gyroscopes noise. Assume the noises follows zero mean Gaussian distribution, with noise covariance matrix $\boldsymbol{Z}=E\left\{\boldsymbol{v}_{k} \boldsymbol{v}_{k}^{\mathrm{T}}\right\}=\left[\begin{array}{cc}\sigma_{a}^{2} \boldsymbol{I}_{3,3} & \mathbf{0}_{3,3} \\ \mathbf{0}_{3,3} & \sigma_{\omega}^{2} \boldsymbol{I}_{3,3}\end{array}\right]$ where $\sigma_{a}^{2}$ and $\sigma_{\omega}^{2}$ respectively represent accelerometers and gyroscopes noise variance.

Since the sensor measurement can be obtained from the joint probability density as:

$$
p\left(\boldsymbol{y}_{n} ; \theta, \mathrm{H}_{i}\right)=\prod_{k \in \Omega_{n}} p\left(\boldsymbol{x}_{k}^{a} ; \theta, \mathrm{H}_{i}\right) p\left(\boldsymbol{x}_{k}^{\omega} ; \theta, \mathrm{H}_{i}\right)
$$

where,

$$
\begin{aligned}
& p\left(\boldsymbol{x}_{k}^{a} ; \theta, \mathrm{H}_{i}\right)=\frac{1}{\left(2 \pi \sigma_{a}^{2}\right)^{3 / 2}} \exp \left\{-\frac{1}{2 \sigma_{a}^{2}}\left\|\boldsymbol{x}_{k}^{a}-\boldsymbol{s}_{k}^{a}(\theta)\right\|^{2}\right\} \\
& p\left(\boldsymbol{x}_{k}^{\omega} ; \theta, \mathrm{H}_{i}\right)=\frac{1}{\left(2 \pi \sigma_{\omega}^{2}\right)^{3 / 2}} \exp \left\{-\frac{1}{2 \sigma_{\omega}^{2}}\left\|\boldsymbol{x}_{k}^{\omega}-\boldsymbol{s}_{k}^{\omega}(\theta)\right\|^{2}\right\}
\end{aligned}
$$

GLRT is determined by the hypothesis $\mathrm{H}_{0}$ if

$$
L_{G}\left(y_{n}\right)=\frac{p\left(\boldsymbol{y}_{n} ; \hat{\theta}_{0}, \mathrm{H}_{0}\right)}{p\left(\boldsymbol{y}_{n} ; \hat{\theta}_{1}, \mathrm{H}_{1}\right)}>\lambda
$$

Where $\lambda$ denotes the threshold. In the eq.(12), $\hat{\theta}_{0}$ and $\hat{\theta}_{1}$ represent the maximum likelihood estimate of the unknown element under the assumptions $\mathrm{H}_{0}$ and $\mathrm{H}_{1}$, respectively. The formula (13) can be simplified as

$$
\begin{aligned}
& L_{G}\left(\boldsymbol{y}_{n}\right)=\exp \left(-\frac{1}{2 \sigma_{a}^{2}} \sum_{k \in \Omega_{n}}\left\|\boldsymbol{x}_{k}^{a}-g \frac{\overline{\boldsymbol{x}}_{k}^{a}}{\left\|\overline{\boldsymbol{x}}_{k}^{a}\right\|}\right\|^{2}-\frac{1}{2 \sigma_{\omega}^{2}} \sum_{k \in \Omega_{n}}\left\|\boldsymbol{x}_{k}^{\omega}\right\|^{2}\right) \\
& T\left(\boldsymbol{y}_{n}\right)=-\frac{2}{N} \ln L_{G}\left(\boldsymbol{y}_{n}\right)=\frac{1}{N} \sum_{k \in \Omega_{n}}\left(\frac{1}{\sigma_{a}^{2}}\left\|\boldsymbol{x}_{k}^{a}-g \frac{\overline{\boldsymbol{x}}_{k}^{a}}{\left\|\overline{\boldsymbol{x}}_{k}^{a}\right\|}\right\|^{2}+\frac{1}{\sigma_{\omega}^{2}}\left\|\boldsymbol{x}_{k}^{\omega}\right\|^{2}\right)
\end{aligned}
$$

$T\left(\boldsymbol{y}_{n}\right)<\lambda$ means that the pedestrian is in a stationary state.

In practice, ZUPT can effectively aid inertial navigation system to remove long-time accumulated errors [5,17-18]. The velocity error of carrier is used as a concept [19-20]. When pedestrians stay static, the MIMU measured velocity is regarded as error to correct the system using Kalman filtering.

The state error vector is defined as

$$
\delta \boldsymbol{X}=\left[\begin{array}{lllll}
\delta \phi^{\mathrm{T}} & \delta \omega^{\mathrm{T}} & \delta r^{\mathrm{T}} & \delta v^{\mathrm{T}} & \delta a^{\mathrm{T}}
\end{array}\right]^{\mathrm{T}}
$$

which respectively represents three-dimensional attitude error, gyro drift, position error, velocity error and accelerometer bias.

The zero-velocity correction Kalman filter model is

$$
\left\{\begin{array}{l}
\delta \boldsymbol{X}_{k}=\boldsymbol{\Phi}_{k} \delta \boldsymbol{X}_{k-1}+\boldsymbol{W}_{k-1} \\
\delta \boldsymbol{Z}_{k}=\boldsymbol{H}_{k} \delta \boldsymbol{X}_{k}+\boldsymbol{N}_{k}
\end{array}\right.
$$

where the state transition matrix is given as 


$$
\begin{gathered}
\boldsymbol{\Phi}_{k}=\left[\begin{array}{ccccc}
\boldsymbol{I}_{3,3} & -\Delta t C_{b k \mid k-1}^{n} & \mathbf{0}_{3,3} & \mathbf{0}_{3,3} & \mathbf{0}_{3,3} \\
\mathbf{0}_{3,3} & \boldsymbol{I}_{3,3} & \mathbf{0}_{3,3} & \mathbf{0}_{3,3} & \mathbf{0}_{3,3} \\
\mathbf{0}_{3,3} & \mathbf{0}_{3,3} & \boldsymbol{I}_{3,3} & \Delta t \boldsymbol{I}_{3,3} & \mathbf{0}_{3,3} \\
\Delta t S\left(f_{k}^{n}\right) & \mathbf{0}_{3,3} & \mathbf{0}_{3,3} & \boldsymbol{I}_{3,3} & \Delta t C_{b k \mid k-1}^{n} \\
\mathbf{0}_{3,3} & \mathbf{0}_{3,3} & \mathbf{0}_{3,3} & \mathbf{0}_{3,3} & \boldsymbol{I}_{3,3}
\end{array}\right] \\
\boldsymbol{S}\left(f_{k}^{n}\right)=\left[\begin{array}{ccc}
0 & -a_{z k}^{n} & a_{y k}^{n} \\
a_{z k}^{n} & 0 & -a_{x k}^{n} \\
-a_{y k}^{n} & a_{x k}^{n} & 0
\end{array}\right]
\end{gathered}
$$

where $\boldsymbol{S}\left(f_{k}^{n}\right)$ is the specific force anti-symmetric matrix, and $\boldsymbol{H}_{k}=\left[\begin{array}{lllll}\mathbf{0}_{3,3} & \mathbf{0}_{3,3} & \mathbf{0}_{3,3} & \boldsymbol{I}_{3,3} & \mathbf{0}_{3,3}\end{array}\right]$

\subsection{The ellipsoidal constraint method}

Each foot are fixedly mounted by a MIMU. For regular human kinematics, the separation distance between the right and left feet cannot be bigger than a quantity known as foot-to-foot maximum separation [8-9]. The maximum step size is a typical feature of pedestrian to walk and can be used to constrain the navigation error [21-22], namely, in addition to using ZUPT to improve the accuracy of pedestrian navigation. In specific, we decompose the constraint into three degrees of freedom and then use the obtained sub-constraints to correct the navigation system. Based on this intuition, we constrain the position estimate of right and left foot-mounted ZUPT-aided INSs.

For two MIMU navigation systems, the $i:$ th $(i \in\{\mathrm{L}, \mathrm{R}\})$ system real state is described as $\boldsymbol{x}_{k}^{i}$ (including position, velocity, and attitude), the estimated state as $\hat{\boldsymbol{x}}_{k}^{i}$ at the time $k$, where $\boldsymbol{x}_{k}^{i} \in \mathbb{R}^{n_{i}}, \hat{\boldsymbol{x}}_{k}^{i} \in \mathbb{R}^{n_{i}}$.

The joint state vector is defined as

$$
\left\{\begin{array}{l}
\boldsymbol{x}_{k} \stackrel{\text { def }}{=}\left[\begin{array}{ll}
\left(\boldsymbol{x}_{k}^{\mathrm{L}}\right)^{\mathrm{T}} & \left(\boldsymbol{x}_{k}^{\mathrm{R}}\right)^{\mathrm{T}}
\end{array}\right]^{\mathrm{T}} \\
\hat{\boldsymbol{x}}_{k} \stackrel{\text { def }}{=}\left[\begin{array}{ll}
\left(\hat{\boldsymbol{x}}_{k}^{\mathrm{L}}\right)^{\mathrm{T}} & \left(\hat{\boldsymbol{x}}_{k}^{\mathrm{R}}\right)^{\mathrm{T}}
\end{array}\right]^{\mathrm{T}}
\end{array}\right.
$$

Here $\hat{\boldsymbol{x}}_{k} \in \mathbb{R}^{m}\left(n_{1}+n_{2}=m\right)$.

Letting the maximum step size of pedestrian be given by $\gamma$, the real displacement difference between the two navigation systems should be less than or equal to $\gamma$.As the leg height subjects to certain constraints, during the pedestrian normal walking state, the positions of the right and left foot can be approximately constrained in a hemisphere ellipsoid (Fig. 1). The position of one foot is constrained within the circle of radius $\gamma$ in the $X O Y$ plane, and is confined within the circle with leg-related radius $h$ in $X O Z$ and $Y O Z$ planes, both centered at the other foot(Fig. 2).

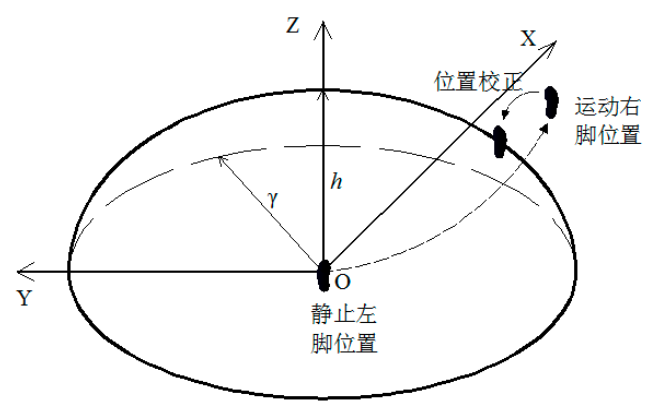

Figure 1: The Ellipsoid constraint schematic $(h<\gamma)$

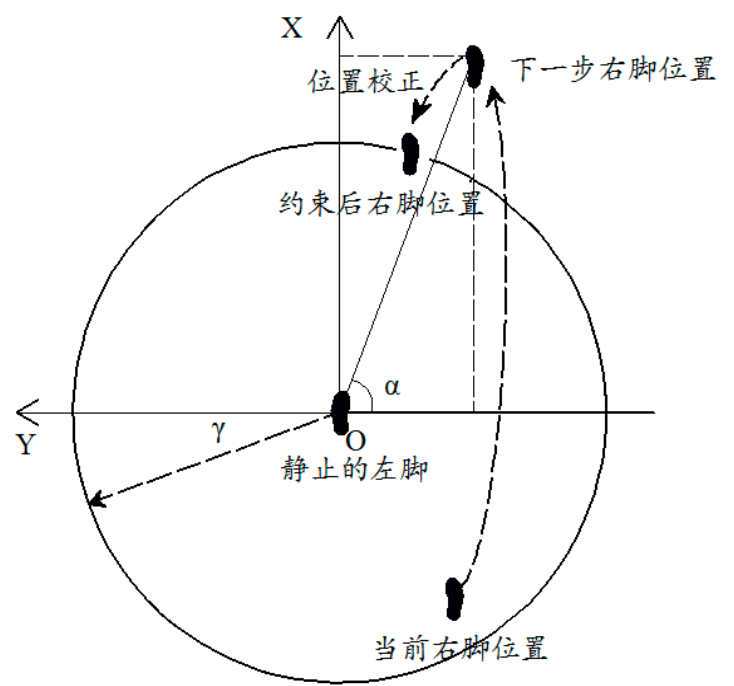

Figure 2: Level constraint for one foot: a circle of radius $\gamma$, centered at the other foot 


$$
\begin{gathered}
\alpha=\arctan \left|\frac{x_{k}^{\mathrm{R}}-x_{k}^{\mathrm{L}}}{y_{k}^{\mathrm{R}}-y_{k}^{\mathrm{L}}}\right| \\
\beta=\arctan \frac{\left|z_{k}^{\mathrm{R}}-z_{k}^{\mathrm{L}}\right|}{\sqrt{\left(y_{k}^{\mathrm{R}}-y_{k}^{\mathrm{L}}\right)^{2}+\left(x_{k}^{\mathrm{R}}-x_{k}^{\mathrm{L}}\right)^{2}}} \\
{\left[\begin{array}{c}
\boldsymbol{L}_{s} \\
\boldsymbol{L}_{h}
\end{array}\right] \cdot x_{k} \leq\left(\begin{array}{l}
\gamma_{x} \\
\gamma_{y} \\
h_{z}
\end{array}\right)}
\end{gathered}
$$

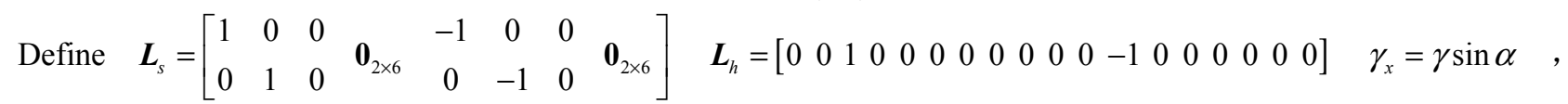
$\gamma_{y}=\gamma \cos \alpha, h_{z}=h \tan \beta$. We assume that two navigation systems attitude is accurate in the current moment when the decompose step size constraint. When $\frac{\left\|\boldsymbol{L}_{s} \cdot \boldsymbol{x}_{k}\right\|^{2}}{\gamma^{2}}+\frac{\left\|\boldsymbol{L}_{h} \cdot \boldsymbol{x}_{k}\right\|^{2}}{h_{z}^{2}}>1$ can constraint the state to be in satisfy $\left\{\boldsymbol{x} \in \mathbb{R}^{n}: \frac{\left\|\boldsymbol{L}_{s} \cdot \boldsymbol{x}_{k}\right\|^{2}}{\gamma^{2}}+\frac{\left\|\boldsymbol{L}_{h} \cdot \boldsymbol{x}_{k}\right\|^{2}}{h_{z}^{2}} \leq 1\right\}$ the state modification is recommended as

$$
\left\{\begin{array}{l}
p\left(\hat{\boldsymbol{x}}_{k}\right) \stackrel{\text { def }}{=} \arg _{x} \min \left(\hat{\boldsymbol{x}}_{k}-\boldsymbol{x}\right)^{\mathrm{T}} \boldsymbol{P}_{k}^{-1}\left(\hat{\boldsymbol{x}}_{k}-\boldsymbol{x}\right) \\
\frac{\left\|\boldsymbol{L}_{s} \cdot \boldsymbol{x}_{k}\right\|^{2}}{\gamma^{2}}+\frac{\left\|\boldsymbol{L}_{h} \cdot \boldsymbol{x}_{k}\right\|^{2}}{h_{z}^{2}} \leq 1
\end{array}\right.
$$

where $\boldsymbol{P}_{k}^{-1}$ denotes the Kalman filter estimated covariance matrix state.

$$
\begin{aligned}
& \text { Define } \boldsymbol{L}=\left[\begin{array}{cccccccc}
1 / \gamma_{x} & 0 & 0 & & -1 / \gamma_{x} & 0 & 0 & \\
0 & 1 / \gamma_{y} & 0 & \mathbf{0}_{3 \times 6} & 0 & -1 / \gamma_{y} & 0 & \mathbf{0}_{3 \times 6} \\
0 & 0 & 1 / h_{z} & 0 & 0 & -1 / h_{z} &
\end{array}\right] \text { and (21) can be written as } \\
& \left\{\begin{array}{l}
p\left(\hat{\boldsymbol{x}}_{k}\right) \stackrel{\text { def }}{=} \arg _{\boldsymbol{x}} \min \left(\hat{\boldsymbol{x}}_{k}-\boldsymbol{x}\right)^{\mathrm{T}} \boldsymbol{P}_{k}^{-1}\left(\hat{\boldsymbol{x}}_{k}-\boldsymbol{x}\right) \\
\boldsymbol{x}_{k}^{\mathrm{T}} \boldsymbol{L}^{\mathrm{T}} \boldsymbol{L} \boldsymbol{x}_{k} \leq 1
\end{array}\right.
\end{aligned}
$$

\section{Experiment}

To compare the performance of the proposed algorithm with the existing in [8](the spheriacl constraint method). Experiments are carried out using two XSENS MTI-G-700 units and the procedure is summarized as follows:

1) In a complex 2-D environment: Some closed trajectory containing straight line path and turning eight times (turning angle: 90 degrees).

2) In a complex 3-D environment: a 6-story staircase, and parts of corridors in the Sheng-Hua building at the Central South University. The walk strats at the first floor and ends at the sixth floor.

3 ) The pedestrian go downstairs from the sixth floor to the first floor.

According to the experimenter gait characteristics, we set $\gamma=0.6$ meters, $h=0.3$ meters, respectively.

In the 2-D closed experiment, the ZUPT aided INS can track the pedestrian feet positions (cf. Fig.3), but the distance of two feet reaches about 6 meters which is unreasonable in the pedestrian navigation. Comparing the three different sets of trajectories, the position estimation information under the ellipsoidal restriction can obtain the position estimates more accurately. Fig. 4 shows the relative positions of the two feet in the $z$ axis direction. Because the $h_{z}$ constrains the altitude difference, so the feet height difference can be reduced near to the true value and the pedestrian location can be more accurate. 

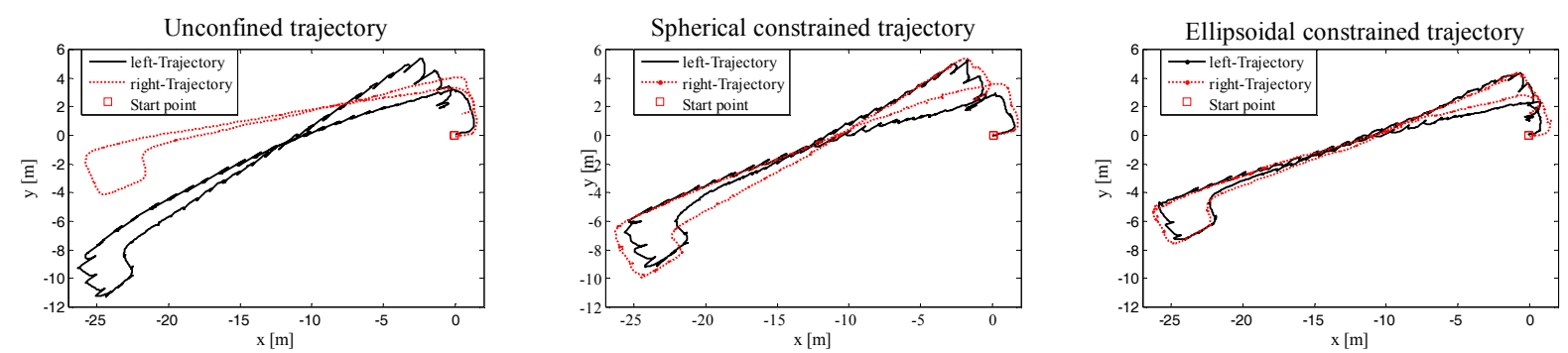

Figure 3: Pedestrian walked with a Closed Path in corridor outside the laboratory
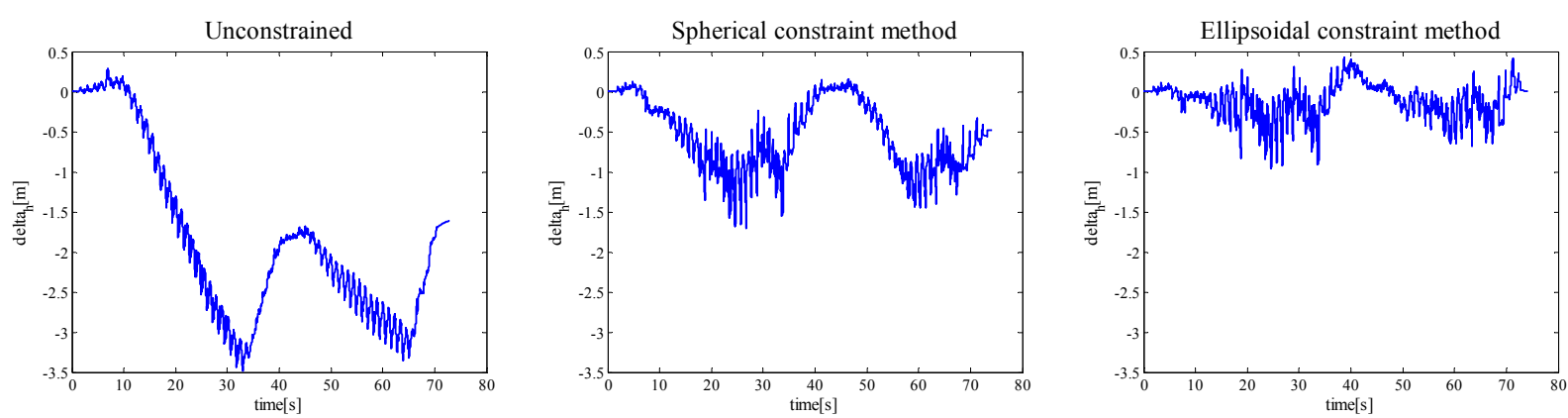

Figure 4: Left and right feet altitude difference in 2-D closed path experiment

In the 3-D upstairs experiment, the result shows the maximum step ellipsoidal constraint method reduces the error accumulation in $z$ axis direction effectively. From the results presented in Fig.5 and Fig.6, we can observe that the proposed method in this paper can reduce the altitude difference of the feet position from 2.6 meters to 0.56 meters. Comparing to the spherical constraint method, the relative positions concentrate between $\mathbf{- 0 . 3}$ to 0.3 meters which are obviously smaller than the spherical confinement results and are more suitable to the characteristics of the feet height on the stairs. Analysising the results obtained in Fig.7 and Fig.8, we observe that the proposed algorithm can track the
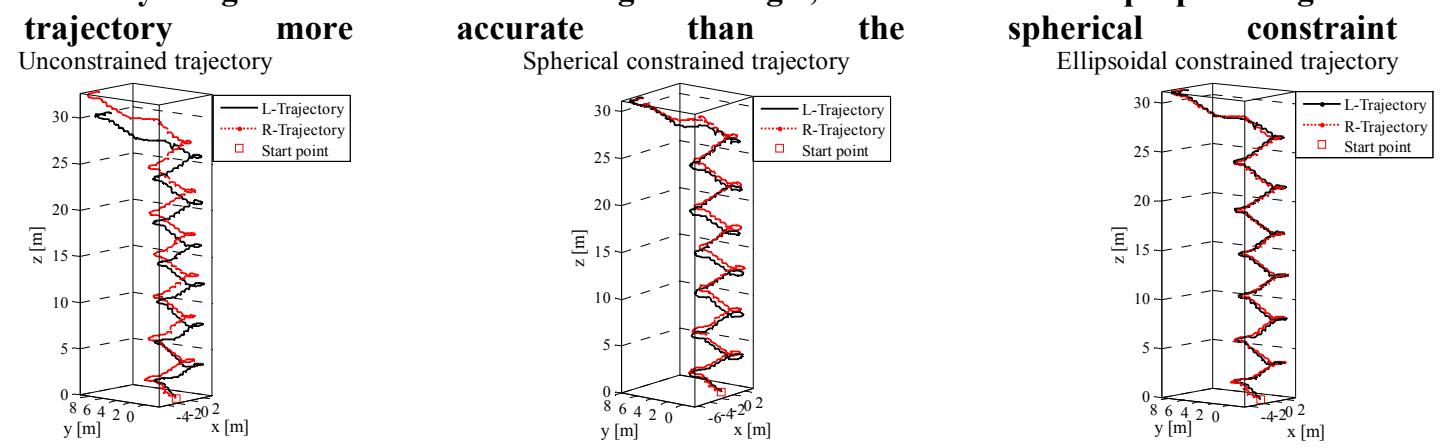
method.

Figure 5: The trajectory of the feet in Upstairs experiment
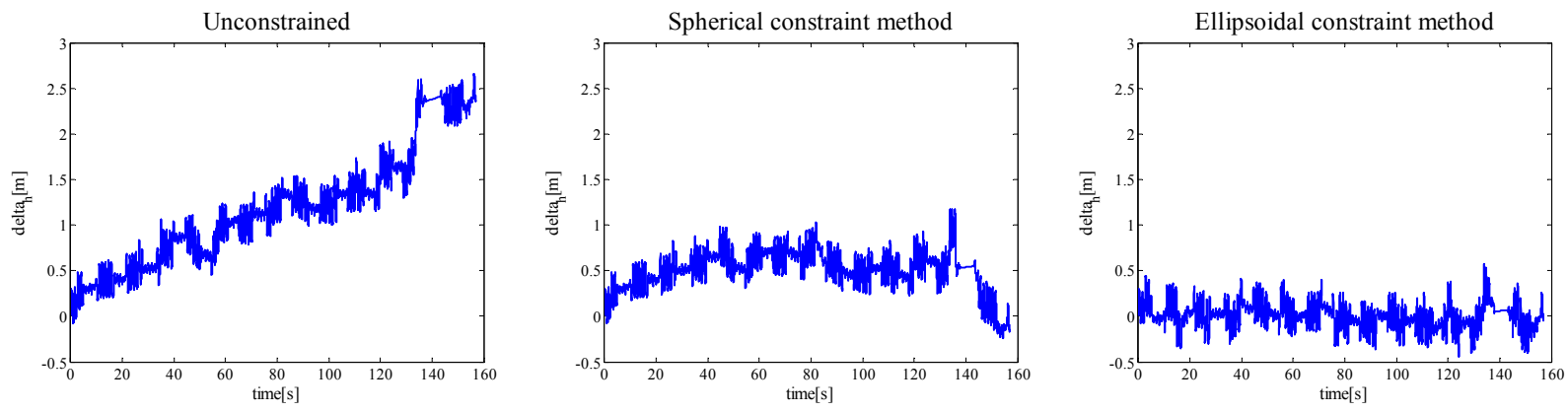

Figure 6: The left and right feet position altitude difference in the Upstairs experiment 

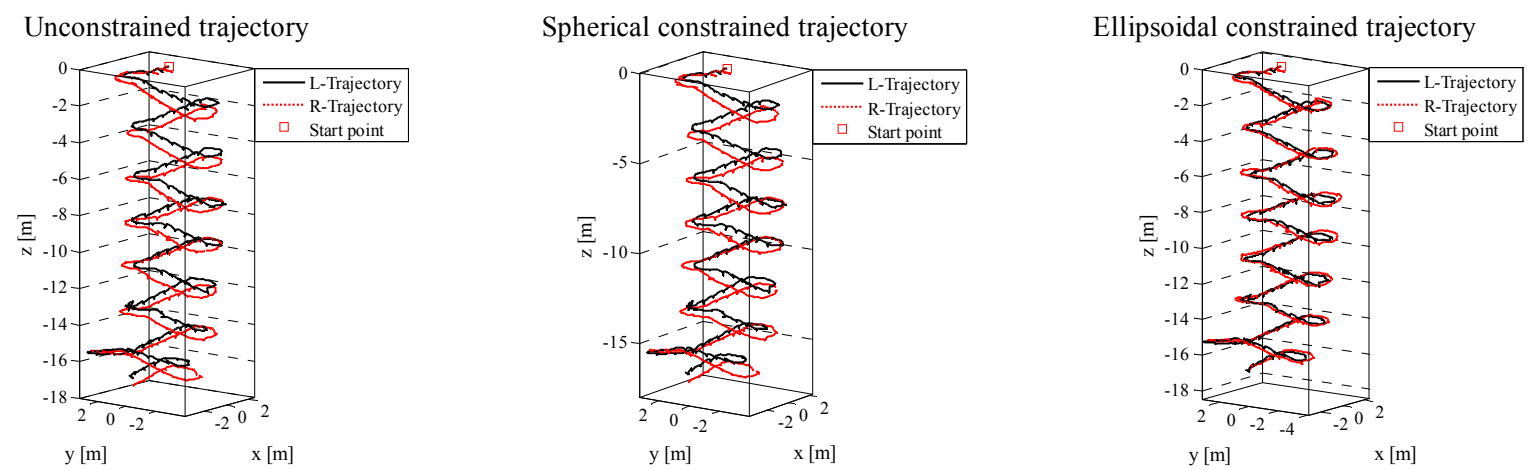

Figure 7: The trajectory of the feet in Downstairs experiment
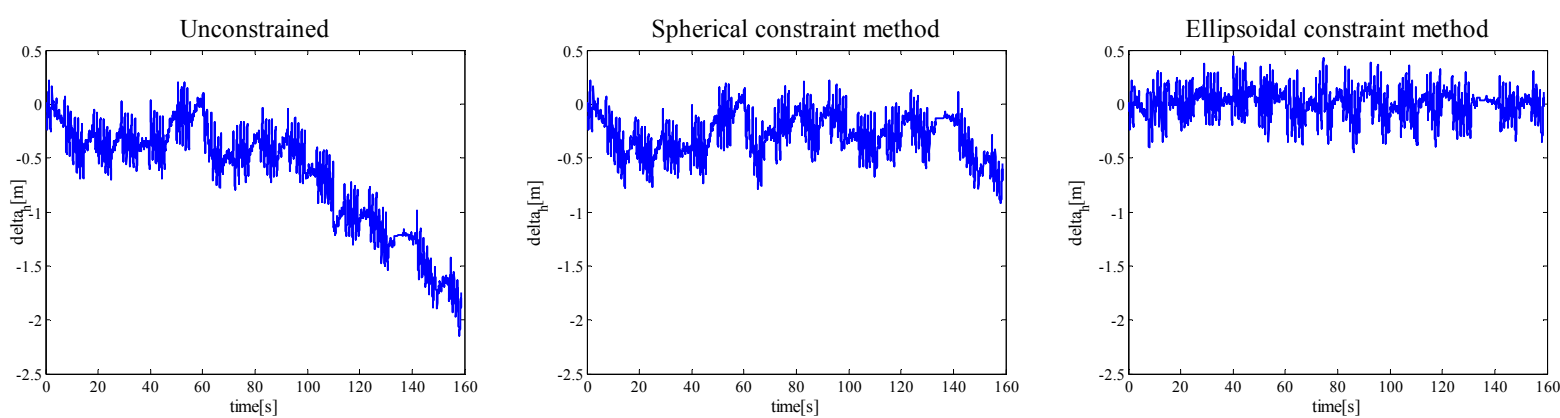

Figure 8: The left and right feet position altitude difference in the Downstairs experiment

\section{Results}

In this paper, we decompose the maximum step along the navigation coordinate system in real-time, and establish an ellipsoidal constraint combining with the actual situation. Each sub-constraint changes with different times, so that the real-time performance of the compensation constraint is improved and the constraint decomposition makes the scheme with step size more specific and accurate. After experimental verification shows that under different experimental path, the proposed ellipsoidal constraint method can effectively correct the position and improve the accuracy of navigation.

\section{ACKNOWLEDGEMENT}

Acknowledgements: The authors gratefully acknowledge the support extended by the National Natural Science Foundation of China. The authors also wish to thank the other staff involved in the project for their contribution and support.

Author Contributions: Wei Shi verificatied algorithm; Yang Wang designed algorithm, simulated and analysised of experimental data; Yuanxin Wu guided the manuscript writing .All authors have read and approved the final manuscript.

Conflicts of Interest: The authors declare no conflict of interest.

\section{References}

[1] Akeila E, Salcic Z, Swain A. Reducing Low-Cost INS Error Accumulation in Distance Estimation Using Self-Resetting[J]. IEEE Transactions on Instrumentation \& Measurement, 2014, 63(1):177-184.

[2] Zhou X C, Chen J X, Dong Y, et al. Pedestrian navigation with foot-mounted inertial sensors in wearable body area networks[C]// Asia-Pacific Signal and Information Processing Association, 2014 Summit and Conference. IEEE, 2014.

[3] Ashkar R, Romanovas M, Goridko V, et al. A low-cost shoe-mounted Inertial Navigation System with magnetic disturbance compensation[C]// International Conference on Indoor Positioning and Indoor Navigation. 2013:1-10.

[4] Jimenez A R, Seco F, Prieto J C, et al. Indoor pedestrian navigation using an INS/EKF framework for yaw drift reduction and a foot-mounted IMU[C]// The Workshop on Positioning Navigation \& Communication. IEEE, 2010:135-143.

[5] Skog I, Händel P, Rantakokko J, et al. Zero-velocity detection in pedestrian navigation systems — an algorithm evaluation[J].

[6] Nilsson J O, Skog I, Handel P, et al. Foot-mounted INS for everybody an open-source embedded implementation[C]// Ieee/ion Plans. 2012:140-145. 
[7] Nilsson J O, Skog I, Händel P. A note on the limitations of ZUPTs and the implications on sensor error modeling[J]. Signal Processing, 2012.

[8] Skog I, Nilsson J O, Zachariah D, et al. Fusing the information from two navigation systems using an upper bound on their maximum spatial separation[J]. Tourism Review, 2012, volume 68(2):14-30(17).

[9] Prateek G V, Girisha R, Hari K V S, et al. Data Fusion of Dual Foot-Mounted INS to Reduce the systematic Heading Drift[C]// International Conference on Intelligent Systems. IEEE, 2013:208-213.

[10] T. Basar. A New Approach to Linear Filtering and Prediction Problems[C]// Wiley-IEEE Press, 2009:35-45.

[11] Welch G, Bishop G. An Introduction to the Kalman Filter[M]. University of North Carolina at Chapel Hill, 1995.

[12] Tully S, Kantor G, Choset H. Inequality constrained Kalman filtering for the localization and registration of a surgical robot[C]// IEEE/RSJ International Conference on Intelligent Robots \& Systems. IEEE, 2011:5147-5152.

[13] Jonghoek Kim, Taeil Suh, Ryu, J. Inequality constrained Kalman filter for Bearing-Only Target Motion Analysis[C]// International Conference on Control, Automation and Systems. 2015:1607-1611.

[14] Dan S, Dan S. Kalman Filter Constraint Tuning for Turbofan Engine Health Estimation[J]. European Journal of Control, 2006, $12(3): 331-343$.

[15] Gupta N, Hauser R. Kalman Filtering with Equality and Inequality State Constraints[J]. Oxford University Computing Laboratory Numerical Analysis Group, 2007.

[16] Simon B D. Kalman filtering with state constraints: a survey of linear and nonlinear algorithms' . http://academic. csuohio.edu / simond / ConstrKF, accessed[J]. 2015.

[17] Skog I, Nilsson J O, Handel P. Evaluation of zero-velocity detectors for foot-mounted inertial navigation systems[C]// International Conference on Indoor Positioning and Indoor Navigation. 2010:1-6.

[18] Skog I, Handel P, Nilsson J O, et al. Zero-Velocity Detection-An Algorithm Evaluation[J]. IEEE Transactions on Biomedical Engineering, 2010, 57(11):2657-2666.

[19] Gao Z Y, De-Sheng L I, Wang Y Z. Combining ZUPT with hybrid particle filter for vehicle MEMS-INS[J]. Electric Machines \& Control, 2010.

[20] Wang Z, Zhao H, Qiu S, et al. Stance phase detection for ZUPT-aided foot-mounted pedestrian navigation system[J]. IEEE/ASME Transactions on Mechatronics, 2015:1-1.

[21] Brand T J, Phillips R E. Foot-to-Foot Range Measurement as an Aid to Personal Navigation[J]. Annual Meeting \& Cigtf Guidance Test Symposium, 2003.

[22] Girisha R, Prateek G V, Hari K V S, et al. Fusing the navigation information of dual foot-mounted zero-velocity-update-aided inertial navigation systems[C]// International Conference on Signal Processing and Communications. IEEE, 2014:1-6.

(C) 2016 by the authors; licensee Preprints, Basel, Switzerland. This article is an open access article distributed under the terms and conditions of the Creative Commons by Attribution (CC-BY) license (http://creativecommons.org/licenses/by/4.0/). 